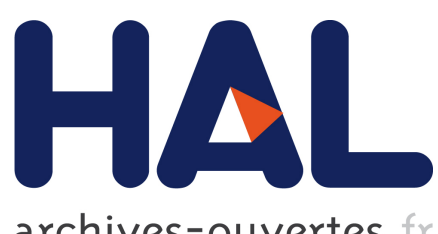

archives-ouvertes

\title{
Exploring different movement sonification strategies for rehabilitation in clinical settings
}

\author{
Frédéric Bevilacqua, Iseline Peyre, Maël Segalen, Pascale Pradat-Diehl, \\ Veronique Marchand-Pauvert, Agnès Roby-Brami
}

\section{To cite this version:}

Frédéric Bevilacqua, Iseline Peyre, Maël Segalen, Pascale Pradat-Diehl, Veronique Marchand-Pauvert, et al.. Exploring different movement sonification strategies for rehabilitation in clinical settings. MOCO '18: 5th International Conference on Movement and Computing, Jun 2018, Genoa Italy, France. pp.1-6, 10.1145/3212721.3212881 . hal-03181442

\section{HAL Id: hal-03181442 \\ https://hal.archives-ouvertes.fr/hal-03181442}

Submitted on 2 Oct 2021

HAL is a multi-disciplinary open access archive for the deposit and dissemination of scientific research documents, whether they are published or not. The documents may come from teaching and research institutions in France or abroad, or from public or private research centers.
L'archive ouverte pluridisciplinaire HAL, est destinée au dépôt et à la diffusion de documents scientifiques de niveau recherche, publiés ou non, émanant des établissements d'enseignement et de recherche français ou étrangers, des laboratoires publics ou privés. 


\section{Exploring different movement sonification strategies for rehabilitation in clinical settings}

Frédéric Bevilacqua

UMR 9912 STMS

Ircam/CNRS/Sorbonne Université

Paris, France

frederic.bevilacqua@ircam.fr

Maël Segalen

UMR STMS Ircam/CNRS/Sorbonne Université

ISIR Sorbonne Université/

CNRS UMR 7222/Inserm U1150

Paris, France

segalen.mael@gmail.com

Véronique Marchand-Pauvert LIB Inserm U1146/CNRS UMR737/

Sorbonne Université

Paris, France

veronique.marchand@upmc.fr
Iseline Peyre

ISIR Sorbonne Université/

CNRS UMR7222/Inserm U1150

LIB Inserm U1146/CNRS UMR737/

Sorbonne Université

Paris, France

peyre@isir.upmc.fr

Pascale Pradat-DiehI

LIB Inserm U1146/CNRS UMR737/

Sorbonne Université

AP-HP HxU Pitié-Salpêtrière Charles Foix

Paris, France

pascale.pradat@psl.aphp.fr

Agnès Roby-Brami

ISIR Inserm U1150/CNRS UMR7222

Sorbonne Université

Paris, France

roby-brami@isir.upmc.fr 


\section{ABSTRACT}

We describe an interactive system that allows for sonifying arm movements. The aim is to support stroke patients going through rehabilitation by providing them with augmented auditory feedback that reacts to their movements. The system is based on IMU sensors (Inertial Measurements Unit) attached to each arm. The movement data are streamed in real-time to a laptop computer that generates various sounds or musical interactions using a program we developed. We tested different types of auditory feedback, each of them using a specific strategy for the sound-movement mapping. The first type of movement-sound mappings is based on direct relationships between the reaching distance and either the pitch of a continuous tone, or the tempo of a regular beat pattern. The second type of mapping is music-oriented: the user movement allows for controlling the tempo of musical pieces. The third type of mapping associates the hand position to specific environmental sounds. We report here on the technical system along with preliminary results in a clinical setting with both post-stroke patients and healthy users.

\section{CCS CONCEPTS}

- Human-centered computing $\rightarrow$ Sound-based input / output; Auditory feedback; Gestural input; Usability testing; Interaction devices;

\section{KEYWORDS}

Sound and Music Computing; Movement and Computing; Rehabilitation; Sonification; Sound; Gesture; Interactive System

\section{ACM Reference Format:}

Frédéric Bevilacqua, Iseline Peyre, Maël Segalen, Pascale Pradat-Diehl, Véronique Marchand-Pauvert, and Agnès Roby-Brami. 2018. Exploring different movement sonification strategies for rehabilitation in clinical settings. In MOCO: 5th International Conference on Movement and Computing, June 28-30, 2018, Genoa, Italy. ACM, New York, NY, USA, 6 pages. https://doi.org/10.1145/3212721.3212881

\section{INTRODUCTION}

The general aim of this research project is to investigate how movement sonification can be put in practice in clinical settings. We use the terms movement sonification to describe a large range of technologies that allow for producing sound and/or music based on human movement [1]. Here, we are currently focusing on stroke patients going through movement rehabilitation.

After a brain lesion due to stroke, the symptoms usually recover spontaneously but partially and inconstantly, and many patients are left with impairment of upper-limb movement, on the opposite side of the lesion. Neuro-rehabilitation is nowadays based on sensorimotor learning principles encouraging 


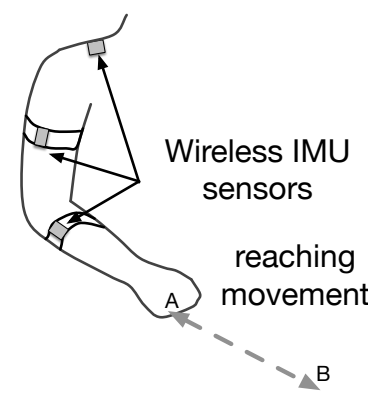

(a)

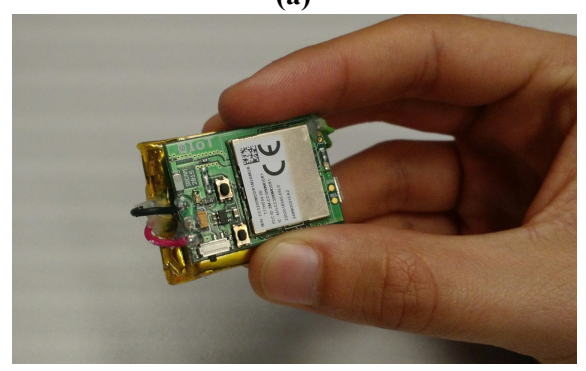

(b)

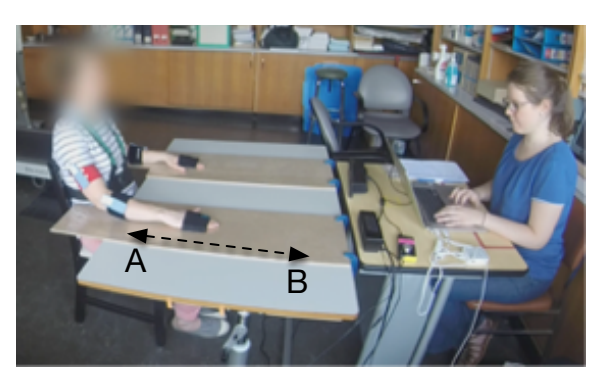

(c)

Figure 1: Experimental setup:

(a) Placement of the IMU sensors

(b) Picture of the IMU sensor (R-IoT)

(c) Picture of the complete setup in clinical setting. The left person is the patient; the right person is the operator patients to move actively in order to foster brain plasticity [6]. It is now established that rehabilitation in the subacute phase should be i) more intense, varied, motivating ii) targeted on the impairments in order to avoid the use of compensations (for example trunk flexion to compensate for the weakness in elbow extension) and iii) prolonged and hopefully followed at home [14].

During rehabilitation tasks, motor learning is facilitated by sensory feedback providing information related to the completion of the task (knowledge of results, KR), and the quality of the performance of the movement (knowledge of performance, KP). Auditory feedback is mostly used in rehabilitation as KR within serious games. In particular, rhythmic feedback has been found to have a positive effect on gait rehabilitation [10]. Continuous sonification is particularly appealing for the rehabilitation of the upper-limb. Sonification can be used to guide the movement direction and intensity in 3D space [3], as well as inducing specific movement qualities.

In contrast to visual feedback shown on a screen, sonification might not add a cognitive load to the motor task, while providing additional sensory input informing on the relative limb position. It can be a substitute to proprioception in the case of associated somatosensory deficit. While some encouraging positive results were observed [12], there are nevertheless fewer studies that investigates how different movement-sound mappings might be used for rehabilitation $[5,7,13]$. In particular the use of simple sonification methods using pitch or rhythm variations is rarely compared to more complex musical interaction or richer interactive sound environments [4].

We report on the development of a movement sonification system specifically designed for clinical settings, which allows us to compare various sonification approaches with patients, the medical staff, as well as valid participants. As this is a work in progress, we present here only the setup along with preliminary results.

\section{GENERAL METHODOLOGY AND SYSTEM}

\section{Experimental design approach}

We followed an iterative approach to include as much as possible the medical staff (principally the occupational therapists), in order to develop a protocol that is grounded in clinical practice. We first observed sessions and methods used by therapists. In parallel, we presented the different tools we had developed previously to the medical staff, from basic sonification systems to gesture-based digital musical instruments ([9], see also http://ismm.ircam.fr). After discussions with both the researchers and the medical staff, we decided to focus on reaching exercises that are routinely performed with patients. Such exercises are often performed on a table, by slowly moving the arm following a segment from A to B, as described in Fig.1. Note also that one of the researchers (author of this poster) is a music therapist, and help in designing the different mapping. 


\section{Real-time movement tracking and sound processing}

We chose to implement a system based on 3 Inertial Measurement Units (IMU), with 3D accelerometers, gyroscope and magnetometers. Each IMU (called R-IoT) transmits data at $200 \mathrm{~Hz}$ through Wi-Fi. Two IMUs are attached to the lower and upper arms, and are used to compute a measure of the reaching distance. A calibration procedure is performed before each measurement session to obtain a normalize distance parameter, used for the sonification. The laptop computer, connected to a soundcard and speakers, operates the sonification using a program written with Max7 (Cycling'74) and the extension MuBu for Max [11]. This library allows for performing signal processing, interactive machine learning as well as sound synthesis such as granular and concatenative synthesis.

\section{Sonifcation strategies}

We decided to implement 3 different sonification paradigms, in order to evaluate how different sounds and musical interactions could either be more efficient or preferred by the users in this context.

\section{(1) Direct sonification}

(a) Pitch: direct relationship between the reaching distance and the pitch. In order to avoid the annoyance of a pure tone, we use granular synthesis in order to vary the pitch of a sample sound (that contains a rich spectrum). The farther the reaching point, the higher the pitch.

(b) Beat: direct relationship between the reaching distance and the tempo of a regular beat pulsation. We used a drum sound, with a regular rhythmic pattern (such as 4 quarter notes). The farther the reaching point, the faster the tempo.

\section{(2) Musical interaction}

In this paradigm, the user can play a melody by moving the arm. All the notes are programmed, so the task consist of activate the progression of the melody (rather than playing the right notes). Two distinct cases were implemented:

(a) Note level: a full forward arm movement triggers four distinct notes. The backward movement triggers the following four notes.

(b) Musical phrase level: a full arm movement enables to continuously 'play' a complete musical phrase, using the 'gesture follower' technique [2].

(3) Environmental sounds exploration

In this paradigm, the reaching movement is divided in three different zones, each one being associated with a specific environmental sound. The reaching movement enables to go through different 'soundscapes', labelled as wind, rain/river and birds. 

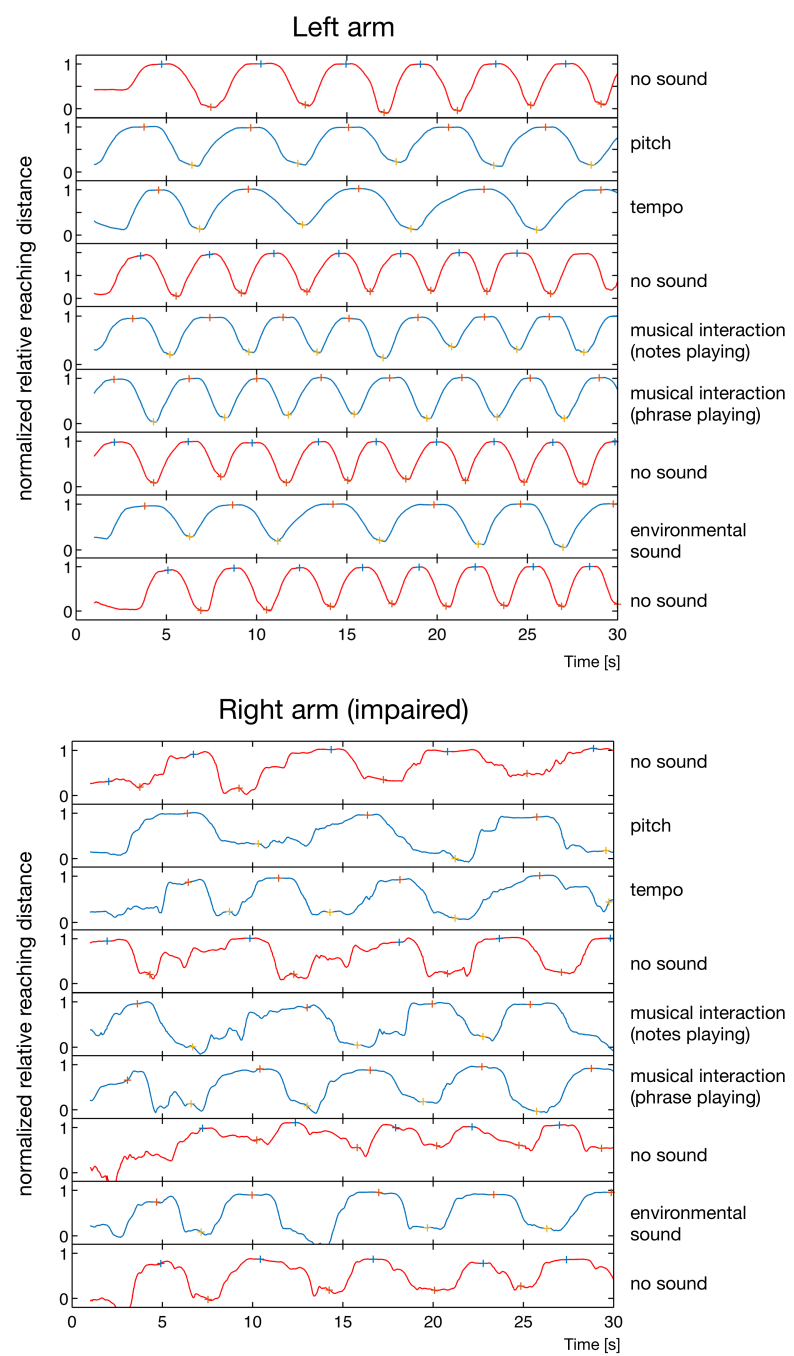

Figure 2: Example of a measurement of the normalized distance measure (one patient).

\section{Protocol}

In the protocol, we ask participants to perform a series of reaching movement for each arm, alternating the use of the different types of sonification described above. A 'no auditory feedback' condition is added at the beginning, the end and between each sonification type (as shown in Fig.2). An amusia evaluation [8] is performed for each participant prior to the experiments, and a complete semistructured interview is performed after the session. All the sensors data are recorded, and all data are kept anonymised. The protocol has been accepted by an ethical committee (CERES: 2016-55).

\section{PRELIMINARY RESULTS AND DISCUSSION}

The data collection is not completed at this point: so far we have investigated 8 post-stroke patients and 7 healthy persons. As an example, we display in Fig.2 the measurements obtained with one post-stroke patient. Differences in the movement speed and fluidity can be clearly seen between the (most) impaired side (right) compared to the other side (left). We can also see that the sonifcation tends to slow the forward-backward movements.

The complete quantitative analysis is out of scope of this extended abstract. Nevertheless, we can already present here some tendency on the preferred type of sonification. During the interviews, we asked the participants to classify their preferred sonification. Among the post-stroke patients, 5/8 preferred the environmental sound exploration, and $3 / 8$ preferred the musical interaction (phrase playing, 2.b). In comparison with the other sonification, the direct mapping (pitch and beat/tempo) was less appreciated than the other ones. Among the healthy subject, a similar trend was observed since 3/7 preferred the environmental sound exploration, 2/7 the musical interaction (phrase playing 2.b.) and 2/7 preferred the other musical interaction (note playing, 2.a). Thus, in all cases, the direct sonification approaches based on pitch or a simple beat pattern, while being very clear perceptually, are not rated as interesting or motivating in this context.

While it is too early to conclude, these preliminary findings point out towards the importance of the overall sound quality and the interaction metaphor to properly implement auditory feedback in clinical settings. As previously discussed by several authors, the motivational aspect of the sound/music might be key in such application.

\section{ACKNOWLEDGMENTS}

The IMU sensors (R-IoT) were developed by Emmanuel Fléty. This work was accomplished within the laboratory of Excellence SMART supported by French state funds managed by the ANR within the Investissements d'Avenir programme under reference ANR-11-IDEX-0004-02 


\section{REFERENCES}

[1] Frédéric Bevilacqua, Eric O. Boyer, Jules Françoise, Olivier Houix, Patrick Susini, Agnès Roby-Brami, and Sylvain Hanneton. 2016. Sensori-Motor Learning with Movement Sonification: Perspectives from Recent Interdisciplinary Studies. Frontiers in Neuroscience 10 (2016), 385. https://doi.org/10.3389/fnins.2016.00385

[2] Frédéric Bevilacqua, Bruno Zamborlin, Anthony Sypniewski, Norbert Schnell, Fabrice Guédy, and Nicolas Rasamimanana. 2010. Continuous Realtime Gesture Following and Recognition. In LNCS. Vol. 5934. Springer Berlin Heidelberg, 73-84.

[3] Eric O. Boyer, Quentin Pyanet, Sylvain Hanneton, and Frédéric Bevilacqua. 2014. Learning Movement Kinematics with a Targeted Sound. In Lecture Notes in Computer Science. Vol. 8905. Springer Verlag, 218-233.

[4] Gaël Dubus and Roberto Bresin. 2013. A systematic review of mapping strategies for the sonification of physical quantities. PloS one 8, 12 (Jan. 2013), e82491. https://doi.org/10.1371/journal.pone.0082491

[5] Hsiu-Yun Hsu, Cheng-Feng Lin, Fong-Chin Su, Huan-Ting Kuo, Haw-Yen Chiu, and Li-Chieh Kuo. 2012. Clinical application of computerized evaluation and re-education biofeedback prototype for sensorimotor control of the hand in stroke patients. Journal of NeuroEngineering and Rehabilitation 9, 1 (09 May 2012), 26. https://doi.org/10.1186/1743-0003-9-26

[6] Tomoko Kitago and John W. Krakauer. 2013. Chapter 8 - Motor learning principles for neurorehabilitation. In Neurological Rehabilitation, Michael P. Barnes and David C. Good (Eds.). Handbook of Clinical Neurology, Vol. 110. Elsevier, 93 - 103. https://doi.org/10.1016/B978-0-444-52901-5.00008-3

[7] Ruth A. Maulucci and Richard H. Eckhouse. 2001. Retraining reaching in chronic stroke with real-time auditory feedback. NeuroRehabilitation 16, 3 (Jan. 2001), 171-82. http://www.ncbi.nlm.nih.gov/pubmed/11790902

[8] Isabelle Peretz, Anne Sophie Champod, and Krista Hyde. 2003. Varieties of Musical Disorders: the Montreal Battery of Evaluation of Amusia. Annals of the New York Academy of Sciences 999, 1 (2003), 58-75. https://doi.org/10.1196/annals 1284.006 arXiv:https://nyaspubs.onlinelibrary.wiley.com/doi/pdf/10.1196/annals.1284.006

[9] Nicolas Rasamimanana, Frederic Bevilacqua, Norbert Schnell, Fabrice Guedy, Emmanuel Fléty, Come Maestracci, Bruno Zamborlin, Jean-Louis Frechin, and Uros Petrevski. 2011. Modular Musical Objects Towards Embodied Control of Digital Music. In Proceedings of the Fifth International Conference on Tangible, Embedded, and Embodied Interaction (TEI '11). 9-12. https://doi.org/10.1145/1935701.1935704

[10] Andrea Schiavio and Eckart Altenmüller. 2015. Exploring Music-Based Rehabilitation for Parkinsonism through Embodied Cognitive Science. Frontiers in Neurology 6 (2015), 217. https://doi.org/10.3389/fneur.2015.00217

[11] Norbert Schnell, Axel Röbel, Diemo Schwarz, Geoffroy Peeters, and Riccardo Borghesi. 2009. MuBu \& Friends - Assembling Tools for Content Based Real-Time Interactive Audio Processing in Max/MSP. In Proceedings of the International Computer Music Conference (ICMC).

[12] Daniel S. Scholz, Sönke Rohde, Nikou Nikmaram, Hans-Peter Brückner, Michael Großbach, Jens D. Rollnik, and Eckart O. Altenmüller. 2016. Sonification of Arm Movements in Stroke Rehabilitation - A Novel Approach in Neurologic Music Therapy. Frontiers in Neurology 7 (2016), 106. https://doi.org/10.3389/fneur.2016.00106

[13] Daniel S. Scholz, Liming Wu, Jonas Pirzer, Johann Schneider, Jens D. Rollnik, Michael Großbach, and Eckart O. Altenmüller. 2014. Sonification as a possible stroke rehabilitation strategy. Frontiers in Neuroscience 8 (2014), 332. https://doi.org/10 3389/fnins.2014.00332

[14] Carolee J. Winstein, Joel Stein, Ross Arena, Barbara Bates, Leora R. Cherney, Steven C. Cramer, Frank Deruyter, Janice J. Eng, Beth Fisher, Richard L. Harvey, Catherine E. Lang, Marilyn MacKay-Lyons, Kenneth J. Ottenbacher, Sue Pugh, Mathew J. Reeves, Lorie G. Richards, William Stiers, Richard D. Zorowitz, and . 2016. Guidelines for Adult Stroke Rehabilitation and Recovery. Stroke (2016). https://doi.org/10.1161/STR.00000000000000098 arXiv:http://stroke.ahajournals.org/content/early/2016/05/04/STR.0000000000000098.full.pdf 